\title{
A martingale central limit theorem without negligibility conditions
}

\section{Robert J. Adler}

\begin{abstract}
We obtain sufficient conditions for the convergence of martingale triangular arrays to infinitely divisible laws with finite variances, without making the usual assumptions of uniform asymptotic negligibility. Our results generalise known results for both the martingale case under a negligibility assumption and the classical (independence) case without such assumptions.
\end{abstract}

\section{Introduction}

The theory of convergence in law of sums of independent random variables has recently been enlarged to include the situation when the summands do not satisfy asymptotic negligibility conditions. In [4] Dvoretzky raised the problem of how these results carry over to the situation when the summands are no longer independent. In [1] this problem was solved for the case when the summands form a martingale difference sequence and the limit law is gaussian, and we now consider the same problem when the limit law is infinitely divisible with finite variance. our result generalises the martingale results of Brown and Eagleson [3] and Dvoretzky [4] (Theorem 2), as well as the sufficiency part of a result of Machis [6] for the independence case without negligibility assumptions.

\section{The limit theorem}

Following [3], consider a double array of random variables, which we take without loss of generality to be a triangular array, whose rows are martingale difference sequences; that is to say, for each $n=1,2, \ldots$,

Received 7 September 1977. 
we have random variables $X_{n I}, \ldots, X_{n n}$ on a probability space $(\Omega, I, P)$, with sub $\sigma$-fields $I_{=n 0} \subset I_{n l} \subset \ldots \subset I_{n n}$ of $\underline{\underline{I}}$ such that $X_{n k}$ is $I_{=n k}$-measurable and $E\left(X_{n k} \mid I_{=n, k-1}^{I}\right)=0$ almost surely for $k=1,2, \ldots, n$. Such arrays are called martingale triangular arrays. Let

$$
\begin{aligned}
& S_{n k}=\sum_{j=1}^{k} x_{n j}, \sigma_{n k}^{2}=E\left(x_{n k}^{2}\right), \\
& \tilde{\sigma}_{n k}^{2}=E\left(x_{n k}^{2} \mid I_{n, k-1}\right) .
\end{aligned}
$$

Let $K(x)$ be a bounded nondecreasing function, which we take as fixed for the remainder of the paper, and let $\sigma^{2}=K(\infty)$. Denote by $G(x)$ the distribution function of the infinitely divisible distribution with variance $\sigma^{2}$ whose characteristic function $\phi(t)$ is given by

$$
\log \phi(t)=\int_{-\infty}^{\infty}\left(e^{i t x}-1-i t x\right) x^{-2} d K(x)
$$

For each $n$ we shall denote by $\left\{G_{n k}, k=1,2, \ldots, n\right\}$ a decomposition of $G$ into $n$ components, each infinitely divisible with finite variance $\sigma_{n k}^{2}$, and we shall denote the spectral function of $G_{n k}$ by $K_{n k} \cdot$ (See [5] for further definitions, and the proof of the existence of such a decomposition for any $n$.) For such a decomposition, put

$$
\Delta_{n k}(x)=P\left(X_{n k} \leq x \mid \stackrel{I}{=-n, k-1}_{n k}\right)-G_{n k}(x) .
$$

THEOREM. Let $\gamma_{n}$ be a bounded sequence of positive real numbers. If there exist a decomposition $\left\{G_{n k}\right\}$ of $G$ of the above form and a sequence of positive numbers $A_{n}$ for which

$$
\gamma_{n}^{-1}\left[A_{n}{ }_{n}+\sup _{k \in \bar{U}_{n}}\left\{1-\left[G_{n k}\left(A_{n}\right)-G_{n k}\left(-A_{n}\right)\right]\right\}\right] \rightarrow 0 \text { as } n \rightarrow \infty \text {, }
$$

where 
(3)

$$
\alpha_{n}=\sup _{k \in \bar{U}_{n}} \sup _{x} E\left|\Delta_{n k}(x)\right|
$$

and $U_{n}$ is the set of values of the index $k$ for which $\sigma_{n k}^{2}<\gamma_{n}$, then in order that $S_{n n}$ converge in low as $n \rightarrow \infty$ to a random variable with distribution function $G$ it is sufficient that the following conditions hold:

$$
\max _{k \in U_{n}} \tilde{\sigma}_{n j}^{2} \stackrel{P}{\longrightarrow} 0 \text { as } n \rightarrow \infty \text {; }
$$

there exists a finite constant $C$ for which

$$
\lim _{n \rightarrow \infty} P\left(\sum_{k \in U_{n}} \tilde{\sigma}_{n j}^{2}>C\right)=0 ;
$$

(6) $\sum_{k \in U} E\left(X_{n k}^{2} I\left(a<X_{n k} \leq b\right) \mid I_{n}, k-1\right)+\sum_{k \in \bar{U}_{n}}\left(K_{n k}(b)-K_{n k}(a)\right)$

$$
\stackrel{P}{\longrightarrow} K(b)-K(a) \text { as } n \rightarrow \infty
$$

for all continuity points $a, b$ of $K$.

REMARKS I. (a) When the martingale differences $X_{n k}$ are actually independent, it is easy to see that the above result contains the sufficiency part of Theorem 1 of [6], by choosing

$$
\gamma_{n}^{3}=\sup _{1 \leq k \leq n} L\left(P\left(X_{n k} \leq x\right), G_{n k}(x)\right),
$$

where $L$ is the Levy metric.

(b) If the martingale triangular array satisfies the conditions of Theorem 1 of [3], then clearly $\sum_{k=1}^{n} \sigma_{n j}^{2}<c$ for all $n$, so that taking $\gamma_{n}=2 C$ ensures that the array also satisfies the conditions of our theorem.

Proof of the theorem. We commence by introducing a new sequence of random variables, $Y_{n k}$. For every $k \in \bar{U}_{n}$ let $Y_{n k}$ have distribution function $G_{n k}$, where the $G_{n k}$ are given in the statement of the theorem, 
and let these be distributed independently of each other and the $\sigma$-field generated by $\bigcup_{k=1}^{n} I_{n k}$. For $k \in U_{n}$ simply set $y_{n k}=X_{n k}$. Now define the sum $R_{n k}$ by

$$
R_{n k}=\sum_{j=1}^{k} Y_{n j}+\sum_{j=k+1}^{n} X_{n j}
$$

We shall first show that, as $n \rightarrow \infty$,

$$
\left|E\left(\exp \left(i t R_{m}\right)\right)-E\left(\exp \left(i t S_{m}\right)\right)\right| \rightarrow 0
$$

so that to prove the theorem we can replace the $X_{n k}$ 's by the $y_{n k}$ 's without any loss of generality. As in equation (15) of [1] it is easy to show that the left hand side of (7) is not greater than

$$
\sum_{k \in \bar{U}_{n}} E\left|E\left(\exp \left(i t Y_{n k}\right)-\exp \left(i t X_{n k}\right) \mid I_{n, k-1}\right)\right|
$$

Each term of ( 8 ) can be written as

(9) $E\left|\int \exp (i t x) d \Delta_{n k}(x)\right|$

$$
\begin{aligned}
& \leq E\left|\int_{-\infty}^{-A} n e^{i t x} d \Delta_{n k}(x)\right|+E\left|\int_{-A_{n}}^{A} e^{i t x} d \Delta_{n k}(x)\right|+E\left|\int_{A_{n}}^{\infty} e^{i t x_{n k}} d \Delta_{n k}(x)\right| \\
& =I_{1}+I_{2}+I_{3} \text {, say. }
\end{aligned}
$$

Consider now each of these terms separately:

$$
\begin{aligned}
& I_{1} \leq E \int_{-\infty}^{-A}\left|d \Delta n_{k}(x)\right| \leq E\left(P\left(X_{n k} \leq-A_{n} \mid \underset{-n, k-1}{I_{-1}}\right)+G_{n k}\left(-A_{n}\right)\right) \\
& \leq \alpha_{n}+2 G_{n k}\left(-A_{n}\right)
\end{aligned}
$$

from (3). Furthermore, from an integration by parts we have

$$
\begin{aligned}
I_{2} & \leq E\left(\left|\int_{-A_{n}}^{A} i t e^{i t x} \Delta_{n k}(x) d x\right|+\mid\left[e^{i t x} \Delta_{n k}(x)\right]_{-A_{n}}^{A} \|\right) \\
& \leq 2 t A_{n} \alpha_{n}+2 \alpha_{n} .
\end{aligned}
$$


But $k \in \bar{U}_{n}$ entails $\sigma_{n k}^{2}>\gamma_{n}$, and since $\sum_{k \in \bar{U}_{n}} \sigma_{n k}^{2} \leq \sigma^{2}$, there are at

most $\sigma^{2} Y_{n}^{-1}$ indices in $\bar{U}_{n}$. Combining this fact, (9)-(11), and a similar bound for $I_{3}$ gives that (8) is not greater than

$$
\sigma^{2} \gamma_{n}^{-1}\left[4 \alpha_{n}+2 t A_{n} \alpha^{\alpha+4} \sup _{k \in U_{n}}\left\{1-\left[G_{n k}\left(A_{n}\right)-G_{n k}\left(-A_{n}\right)\right]\right\}\right]
$$

which, under the conditions of the theorem, tends to zero as $n \rightarrow \infty$. This establishes (7).

Thus we now need only consider the convergence of $R_{r n}$. By Theorem 2.3 of [2] we need only show that for any subsequence $\left\{n^{\prime}\right\}$ there exists a further subsequence $\left\{n^{\prime \prime}\right\}$ along which convergence occurs. Thus, without any loss of generality, we may assume that

$$
\sum_{k \in \bar{U}_{n}} K_{n k}(b)-K_{n k}(a) \rightarrow K^{(1)}(b)-k^{(1)}(a)
$$

where $K^{(1)}$ is a nondecreasing function dominated by $K$. (The existence of such a function follows from Helly's first theorem.) This condition is of course equivalent to the condition that $\sum_{k \in \bar{U}_{n}} Y_{n k}$ converge in a law to

a random variable with characteristic function $\phi^{(1)}(t)$, given by

$$
\log \phi^{(1)}(t)=\int_{-\infty}^{\infty}\left(e^{i t x}-1-i t x\right) x^{-2} d K(1)(x)
$$

We now define functions $K^{(2)}$ and $\phi^{(2)}$ by

$$
K^{(1)}(x)+K^{(2)}(x)=K(x), \phi^{(1)}(t) \phi^{(2)}(t)=\phi(t) .
$$

Then it follows from (6) and (12) that, as $n \rightarrow \infty$,

$$
\sum_{k \in U} E\left(x_{n k}^{2} I\left(a<x_{n k} \leq b\right) \mid \stackrel{I}{=}, k-1_{n}\right) \stackrel{P}{\rightarrow} K^{(2)}(b)-K^{(2)}(a) \text {. }
$$

If we now apply the same type of argument as on pages 52-53 of [1] (that is, we form a new array from the $\left.y_{n k}, k \in U_{n}\right)$ it follows from Theorem 1 
of [3] that $\sum_{k \in U_{n}} Y_{n k}=\sum_{k \in U_{n}} X_{n k}$ converges in law to the random variable with characteristic function $\phi^{(2)}(t)$. Combining this with (12)-(14) completes the proof of the theorem.

REMARKS 2. (a) We note that as in [3] the assumption that the rows of the triangular array form a martingale difference sequence is not essential. It is a trivial matter to relax this condition as in Theorem 2 of [1] so that in effect our result also generalises Theorem 2 of [4].

(b) It is of interest to obtain sharper forms of (2) when the limit distribution has a simple form. For example, if the limit law is that of a zero mean, unit variance, gaussian random variable, then (2) becomes

$$
\gamma_{n}^{-1} \alpha_{n}\left(\log \alpha_{n}^{-1}\right)^{\frac{1}{2}}+0 \text { as } n \rightarrow \infty \text {. }
$$

This follows simply from (2) by choosing $A_{n}=\left(2 \log \alpha_{n}^{-1}\right)^{\frac{1}{2}}$, and then noting that since each $G_{n k}$ can be taken as the distribution function of a gaussian random variable with zero mean and variance $\sigma_{n k}^{2} \leq 1$, we can use the well known inequality (for large $x$ )

$$
\Phi(-x)=1-\Phi(x) \leq\left(x e^{-\frac{1}{2} x^{2}}\right)^{-1},
$$

where $\Phi$ is the standard gaussian distribution function, to bound the terms in (2).

Similarly, when the limit law is Poisson, each $G_{n k}$ can be taken as Poisson with parameter $\sigma_{n k}^{2} \leq \sigma^{2}$. Thus, using the upper tail bound for this distribution given by

$$
1-G_{n k}\left(A_{n}\right) \leq\left(\sigma_{n k}^{2}\right)^{A_{n}} /\left(A_{n}\right) !,
$$

where $\left\{A_{n}\right\}$ is now taken to be a sequence of positive numbers increasing to infinity, we can apply Stirling's approximation to see that 


$$
1-G_{n k}\left(A_{n}^{\prime}\right) \leq \text { constant }\left(\sigma^{2} e^{1} A_{n}^{-1}\right)^{A} n^{+\frac{1}{2}} \text {, }
$$

which will tend to zero as $n \rightarrow \infty$. Hence, in the Poisson case, (2) can be very simply written as

$$
\gamma_{n}^{-1} \alpha_{n}^{A}{ }_{n} \rightarrow 0 \text { as } n \rightarrow \infty \text {, }
$$

for any integer sequence $\left\{A_{n}\right\}$ for which $A_{n} \rightarrow \infty$ as $n \rightarrow \infty$.

\section{References}

[1] R.J. Adler and D.J. Scott, "Martingale central limit theorems without uniform asymptotic negligibility", Bulz. Austral. Math. Soc. 13 (1975), 45-55.

[2] Patrick Billingsley, Convergence of probability measures (John Wiley \& Sons, New York, London, Sydney, Toronto, 1968).

[3] B.M. Brown and G.K. Eagleson, "Martingale convergence to infinitely divisible laws with finite variances", Trans. Amer. Math. Soc. 162 (1971), 449-453.

[4] Aryeh Dvoretzky, "Central limit theorems for dependent random variables", Actes du Congrès International des Mathématiciens, 1970, Volume 2, 565-570 (Gauthier-Villars, Paris, 1971).

[5] B.V. Gnedenko and A.N. Kolmogorov, Limit distributions for sums of independent random variables (translated by K.L. Chung. AddisonWesley, Cambridge, Massachusetts, 1954).

[6] Yu Yu Machis, "Non-classical limit theorems", Theor. Probability AppZ. 16 (1971), 175-182.

Commonwealth Scientific and Industrial Research Organization, Division of Mathematics and Statistics, Sydney, New South Wales. 